\title{
Separation of solar radio bursts in a complex spectrum
}

\author{
Hana Mészárosová ${ }^{1}$, Ján Rybák ${ }^{2}$, Marian Karlický ${ }^{1}$, \\ and Karel Jiřička ${ }^{1}$ \\ ${ }^{1}$ Astronomical Institute of the Academy of Sciences, \\ CZ-25165 Ondřejov, Czech Republic \\ email: hana@asu.cas.cz \\ ${ }^{2}$ Astronomical Institute, Slovak Academy of Sciences, \\ SK-05960 Tatranská Lomnica, Slovak Republic \\ email: choc@ta3.sk
}

\begin{abstract}
Radio spectra, observed during solar flares, are usually very complex (many bursts and fine structures). We have developed a new method to separate them into individual bursts and analyze them separately. The method is used in the analysis of the $0.8-2.0 \mathrm{GHz}$ radio spectrum of the April 11, 2001 event, which was rich in drifting pulsating structures (DPSs). Using this method we showed that the complex radio spectrum consists of at least four DPSs separated with respect to their different frequency drifts $\left(-115,-36,-23\right.$, and $\left.-11 \mathrm{MHz} \mathrm{s}^{-1}\right)$. These DPSs indicate a presence of at least four plasmoids expected to be formed in a flaring current sheet. These plasmoids produce the radio emission on close frequencies giving thus a mixture of superimposed DPSs observed in the radio spectrum.
\end{abstract}

Keywords. Sun: corona, Sun: flares, Sun: radio radiation, Sun: oscillations

\section{Introduction}

The drifting pulsating structures (Karlický 2004, Reiner et al. 2008) observed at the beginning of the eruptive solar flares have been found to be radio signatures of the plasmoid ejection (Karlický \& Odstrčil 1994, Ohyama \& Shibata 1998, Karlický et al. 2002). Based on the MHD numerical simulations, Kliem et al. (2000) suggested that the drifting pulsation structure is generated by superthermal electrons, trapped in the magnetic island (plasmoid) in the bursty regime of the magnetic field reconnection. The global slow negative frequency drift of the structure was explained by a plasmoid propagation upwards in the solar corona towards lower plasma densities. This model of DPSs was further developed and verified in the papers by Karlický \& Bárta (2007), Bárta et al. (2008), Karlický et al. (2010).

\section{Observations and analysis}

Observed radio spectra usually consist of many radio bursts and fine structures superimposed in the same time and frequency intervals. Therefore, it would be highly desirable to separate these individual radio features and analyze them separately. For this purpose we developed a new method (Mészárosová et al. 2010) that is able to separate the bursts and fine structures according to their frequency widths and temporal scales. The method is based on the wavelet analysis technique. In order to present capabilities of our method we have tested it using artificial radio spectra (Mészárosová et al. 2010). 

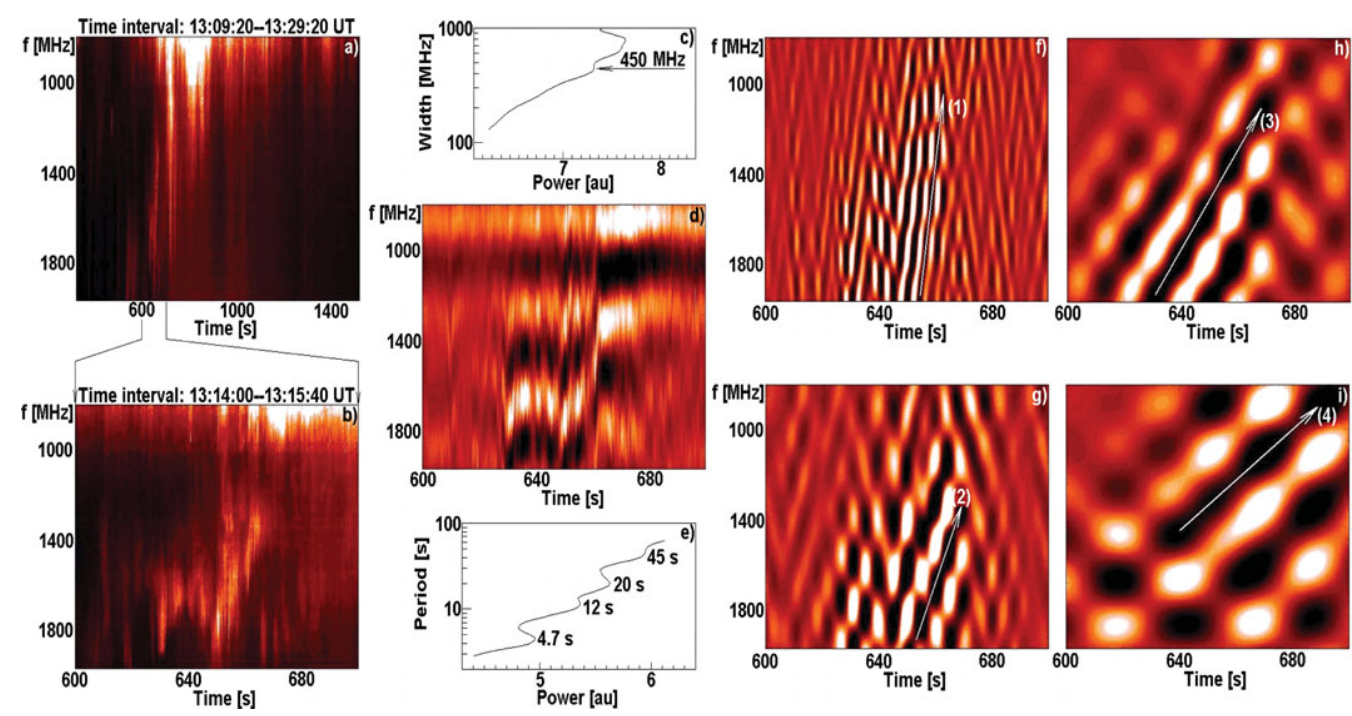

Figure 1. Separation of DPSs in the radio dynamic spectrum of the April 11, 2001 event: (a) original radio spectrum with DPSs, (b) part of the original radio spectrum with DPSs, (c) averaged global spectrum (ASf) with a peak for the frequency width $=450 \mathrm{MHz}$, (d) filtered radio spectrum in the frequency width range $=350-500 \mathrm{MHz}$, (e) averaged global spectrum (ASt) made from the spectrum in the panel $d$ (f) separated DPSs in the period range 3-6 s, (g) separated DPSs in the period range 6-15 s, (h) separated DPSs in the period range 15-29 $\mathrm{s}$, and (i) separated DPSs in the period range 29-52 s. Arrows in panels show frequency drifts of DPSs. The positive and negative parts of amplitudes (in the relation to their mean values) are in white and black colors, respectively (panels $d$ and $f-i$ ).

In the present paper we applied this method on the $0.8-2.0 \mathrm{GHz}$ radio spectrum observed during the April 11, 2001 event (GOES M2.3, NOAA AR9415) by the Ondřejov radiospectrograph (Jiřička et al. 1993). We analyzed this spectrum in the time interval 13:09:20-13:29:20 UT (i.e. $1200 \mathrm{~s}$ ) and in the frequency range 0.8-2.0 GHz (see Figure 1, panel $a$ ). Its time resolution is $0.1 \mathrm{~s}$. As an example of our analysis, we present here the analysis of the spectrum in the time subinterval 13:14:00-13:15:40 UT. As can be seen here, this part of the radio spectrum consists of several superimposed DPSs. We used the same method as in the paper by Mészárosová et al. (2010). We took the spectrum (Figure 1, panel $b$ ) as an input data set for the separation according to frequency widths of DPSs. Then we computed the averaged wavelet spectrum (ASf, panel $c$ ). It shows a peak for the frequency width $450 \mathrm{MHz}$. It means that the most of DPSs in the radio spectrum (panel $b$ ) have the frequency width of about $450 \mathrm{MHz}$. Therefore, we computed a new filtered radio spectrum in the frequency widths range $350-500 \mathrm{MHz}$, where the values 350 and $500 \mathrm{MHz}$ correspond to local minima around the peak at $450 \mathrm{MHz}$. This filtered spectrum is presented in the panel $d$. Then, the data set in panel $d$ became the input data for the separation according to temporal periods of DPSs. The averaged global spectrum (ASt) shows four peaks (panel $e$ ) for the periods $P=4.7,12,20$, and $45 \mathrm{~s}$. It means that the DPSs with frequency width of about $450 \mathrm{MHz}$ have the characteristic periods $4.7,12,20$, and $45 \mathrm{~s}$. Then, using the inverse wavelet transform we computed the final spectra filtered in the period ranges 3-6 s (panel $f$ ), 6-15 s (panel $g$ ), 15-29 s (panel $h$ ), and 29-52 s (panel $i$ ) where these range values correspond to local minima around the period peaks in panel $e$. The positive and negative parts of amplitudes are in white and black colors, respectively (panels $d$ and $f-i$ ). This type of displaying makes possible to see individual DPSs in very good contrast. 
At least four different frequency drifts can be recognized $\left(-115,-36,-23\right.$, and $-11 \mathrm{MHz} \mathrm{s}^{-1}$, see the arrows $1,2,3$, and 4 in the panels $f, g, h$, and $i$, respectively). It means that at least four DPSs are present in the complex radio spectrum observed in the April 11, 2001 event. We have obtained similar results also for other selected subintervals during the time interval 13:15:40-13:29:20 UT.

\section{Conclusions}

Using new method (Mészárosová et al. 2010), the complex spectrum observed during the April 11, 2001 flare with many drifting pulsating structures (DPSs) was analyzed. We found that the characteristic bandwidth of these DPSs is about $450 \mathrm{MHz}$ in whole time interval under study (13:09:20-13:29:20 UT, Figure 1, panel $a)$. The DPSs in time subinterval (13:14:00-13:15:40 UT, panel $b$ ) revealed the frequency drifts $-115,-36,-23$, and $-11 \mathrm{MHz} \mathrm{s}^{-1}$, respectively (panels $f-i$ ). The DPSs of the others time subintervals (13:15:40-13:29:20 UT) show similar frequency drifts.

Usually only one DPS is observed as a signature of the ejection of one dominant plasmoid. However, in the flaring current sheet a series of plasmoids of different sizes can be formed (Shibata \& Tanuma 2001). If these plasmoids produce the radio emission on close frequencies then on the radio spectrum we can see a mixture of different and superimposed DPSs. The radio spectrum of the April 11, 2001 event shows such superimposed DPSs. Our analysis of this spectrum revealed that it consists of at least four different DPSs (panels $f-i$ ), which means that at least four plasmoids (magnetic islands) were generated in the flaring current sheet. But the velocities of these plasmoids, oriented in the upward direction in the solar atmosphere, differ.

\section{Acknowledgements}

H. M., M. K., and K. J. acknowledge support from the Grant IAA300030701 of the Academy of Sciences of the Czech Republic and the research project AVOZ10030501 of the Astronomical Institute AS CR. The work of J. R. was partly supported by the Slovak Grant Agency VEGA (project 2/0064/09). The program of mobility between the academies of the Czech Republic and Slovakia is also acknowledged. The wavelet analysis was performed using the software based on tools provided by C. Torrence and G. P. Compo at http://paos.colorado.edu/research/wavelets.

\section{References}

Bárta, M., Karlický, M., \& Žemlička, R. 2008, Solar Phys., 253, 173

Jiřička, K., Karlický, M., Kepka, O., \& Tlamicha, A. 1993, Solar Phys., 147, 203

Karlický, M. \& Odstrčil, D. 1994, Solar Phys., 155, 171

Karlický, M., Fárník, F., \& Mészárosová, H. 2002, A\&A, 395, 677

Karlický, M. 2004, A\&\&A, 417, 325

Karlický, M. \& Bárta, M. 2007, A\&̛A, 464, 735

Karlický, M., Bárta, M., \& Rybák, J. 2010, A\&̇A, 514, A28

Kliem, B., Karlický, M., \& Benz, A. O. 2000, A\&A, 360, 715

Mészárosová, H., Rybák, J., \& Karlický, M. 2010, Aछ̈A, submitted

Ohyama, M. \& Shibata, K. 1998, ApJ, 499, 934

Reiner, M. J., Klein, K. L., Karlický, M., et al. 2008, Solar Phys., 249, 337

Shibata, K. \& Tanuma, S. 2001, Earth, Planets and Space, 53, 473 